\title{
Die meisten sterben an Krebs
}

\begin{abstract}
Niederländische, belgische und deutsche Palliativmediziner werteten für die meisten EU-Länder die Daten von Eurostat aus, dem statistischen Amt der Europäischen Union, um die Sterbeziffer des Jahres 2007 bei Krebs und anderen chronischen Erkrankungen in Europa zu analysieren. Etwa 495 Mllionen (Mio.) Menschen lebten 2007 in der EU. Von insgesamt 4,8 Mio. Todesfällen waren 2,02 $(42,2 \%)$ auf die Folgen von Krebs oder anderen chronischen Erkrankungen zurückzuführen: Den Hauptanteil machten die Krebskrankheiten aus (26,3\%), gefolgt von Herzinsuffizienz (5,7\%), chronischen Atemwegserkrankungen (3\%), Diabetes $(2,2 \%)$, Leberversagen $(1,8 \%)$ und De-
\end{abstract}

\section{kurz notiert}

Forschungsstandort Deutschland

Bakterielles Toxin initiiert Zellsuizid +++ Ein Ansatz für eine onkologische Therapie? Das Forschungsteam um Klaus Aktories vom Institut für Experimentelle und Klinische Pharmakologie und Toxikologie sowie dem Centre for Biological Signalling Studies (BIOSS) der Universität Freiburg hat den Wirkmechanismus eines bakteriellen Toxins aufgeklärt und gezeigt, dass dieser Giftstoff den Selbstmord von Zellen auslöst [Guttenberg G et al. J Biol Chem. 2012;287(30): 24929-40]. Derzeit untersucht das Freiburger Team, ob das TpeL-Toxin in der Lage ist, Krebs erzeugende Ras-Varianten zu inaktivieren. So könnte es möglich werden, Krebszellen mithilfe des bakteriellen Toxins zum Selbstmord zu führen

Neuroblastom-Gen entdeckt +++ Erstmals ist es einer internationalen Forschergruppe gelungen, die krebsauslösende Wirkung eines bestimmten Gens (Lin28b) bei der Neuroblastom-Entstehung aufzuklären. Federführend in diesem Forschungsprojekt ist Johannes $\mathrm{H}$. Schulte vom Zentrum für Kinder- und Jugendmedizin des Universitätsklinikums Essen. Die Forschergruppe um Schulte konnte nachweisen, dass es sich bei Lin28b wirklich um ein Onkogen handelt, das Tumoren auslösen kann [Molenaar JJ et al. Nat Genet. 2012 Oct 7. doi: 10.1038/ng.2436. (Epub ahead of print)]. Zudem zeigten sie, dass Lin28b für eine zielgerichtete Neuroblastom-Therapie wichtig ist. Geplant ist, Substanzen zu entwickeln, die Lin28b hemmen können. menz (1,6\%). Basierend auf den Totenscheinen betrug die nicht bereinigte Sterbeziffer für Krebs und andere chronische Erkrankungen 409/100.000 EU-Einwohner, für akute Erkrankungen 561. Besonders hoch waren die Ziffern in Bulgarien (594), Ungarn (503) und Dänemark (474). Eher im Mittelfeld befanden sich etwa Deutschland (442), Schweden (417) und die Niederlande (416). Die niedrigsten Sterbeziffern in Verbindung mit Krebs und anderen chronischen Erkrankungen wurden in Zypern (275) und Irland (278) verzeichnet. Laut den Eurostat-Daten lagen die krankheitsbezogenen Sterbeziffern in der Gruppe der Menschen über 65 Jahre deutlich höher - bei 1.783 pro
100.000 Personen. Die Palliativmediziner um Jeroen Hasselaar, Nijmegen, Niederlande, wagten zudem eine Vorhersage, wie groß der Anteil der schmerzgeplagten Patienten in fortgeschrittenem Erkrankungsstadium sein wird. Basierend auf einer Studie aus dem Jahr 2006, in der unter anderem die Symptomverteilung bei Patienten mit Krebs, analysiert wurde, sei zu erwarten, dass 441.000 bis 1,210 Millionen Patienten mit Krebs eine Schmerztherapie brauchen werden. Dem Datenvergleich zufolge sei zudem damit zu rechnen, dass pro Jahr bei 126.000 bis 882.000 Krebspatienten ein Atemnotsyndrom auftreten wird.

Peter Leiner

Hasselaar J et al. The burden of non-acute dying on society: dying of cancer and chronic disease in the European Union. BMJ Supportive \& Palliative Care 2012, online 28. August; doi: 10.1136/ bmjspcare-2011-000162

\section{Bei Prostatakrebspatienten auch auf den Lebensstil achten}

Die Einführung des PSA-Screenings hat einer US-amerikanischen Untersuchung zufolge einen deutlichen Einfluss auf die spezifischen Mortalitätsraten - sowohl in Schweden als auch in den USA. Grundlage der Untersuchung waren die populationsbasierten Daten des Schwedischen Krebsregisters von 1961 bis 2008 sowie des amerikanischen SEER (Suirveillance, Epidemiology, and Results)-Programms von 1973 bis 2008 . Von den schwedischen Prostatakarzinompatienten starben insgesamt $52 \%$ am Prostatakarzinom, $17 \%$ an ischämischer Herzkrankheit und $6 \%$ an anderen Tumoren. In den USA starben $30 \%$ am Prostatakrebs $24 \%$ an ischämischer Herzkrankheit und $15 \%$ an anderen Krebserkrankungen.

Die kumulative 5-Jahres-Inzidenz für einen krankheitsspezifischen Tod infolge Prostatakarzinom ist in den USA in den vergangenen Jahrzehnten deutlich gesunken. Während von den amerikanischen Männern, deren Prostatakrebs in den 1970 er-Jahren diagnostiziert worden war, noch etwa $24 \%$ die kommenden fünf Jahre nicht überlebten, waren dies bei einer Diagnosestellung in den 1990ern nur noch $8 \%$. Die Rate für die ischämische Herzkrankheit als Todesursache bei dieser Patientengruppe reduzierte sich in diesem Zeitraum von 12 auf $6 \%$. Bei den Schweden ist die kumulative 5-JahresInzidenz für einen Tod infolge Prostatakarzinom von $41 \%$ bei Diagnosestellung in den 1960er-Jahren auf $25 \%$ gesunken, wenn die Krankheit in den 1990er-Jahren entdeckt wurde. Die 5-Jahres-Inzidenz für die ischämische Herzkrankheit als Todesursache der Schweden hat sich vergleichsweise wenig verändert.

Sowohl in Schweden als auch in den USA ist das Risiko, an einem Prostatakarzinom zu versterben, deutlich zurückgegangen. Daher sollte der ischämischen Herzerkrankung als Todesursache mehr Beachtung geschenkt werden. Lebensstilveränderungen könnten dieses Risiko verringern und möglicherweise auch das Risiko aufgrund anderer Tumoren $\mathrm{zu}$ versterben senken, so die Autoren.

Christine Starostzik

Epstein MM et al. Temporal Trends in Cause of Death Among Swedish and US Men with Prostate Cancer. J Natl Cancer Inst. 2012;104(17):1335-42 